\title{
Exploiting the Causal Tensor Network Structure of Quantum Processes to Efficiently Simulate Non-Markovian Path Integrals
}

Jørgensen, Mathias Rønnow; Pollock, Felix A.

Published in:

Physical Review Letters

Link to article, DOI:

10.1103/physrevlett.123.240602

Publication date:

2019

Document Version

Publisher's PDF, also known as Version of record

Link back to DTU Orbit

Citation (APA):

Jørgensen, M. R., \& Pollock, F. A. (2019). Exploiting the Causal Tensor Network Structure of Quantum Processes to Efficiently Simulate Non-Markovian Path Integrals. Physical Review Letters, 123(24), [240602]. https://doi.org/10.1103/physrevlett.123.240602

\section{General rights}

Copyright and moral rights for the publications made accessible in the public portal are retained by the authors and/or other copyright owners and it is a condition of accessing publications that users recognise and abide by the legal requirements associated with these rights.

- Users may download and print one copy of any publication from the public portal for the purpose of private study or research.

- You may not further distribute the material or use it for any profit-making activity or commercial gain

- You may freely distribute the URL identifying the publication in the public portal 


\title{
Exploiting the Causal Tensor Network Structure of Quantum Processes to Efficiently Simulate Non-Markovian Path Integrals
}

\author{
Mathias R. Jørgensen ${ }^{1, *}$ and Felix A. Pollock $\oplus^{2, \dagger}$ \\ ${ }^{1}$ Department of Physics, Technical University of Denmark, 2800 Kongens Lyngby, Denmark \\ ${ }^{2}$ School of Physics and Astronomy, Monash University, Clayton, Victoria 3800, Australia
}

\begin{abstract}
(Received 24 April 2019; revised manuscript received 21 September 2019; published 9 December 2019)
In the path integral formulation of the evolution of an open quantum system coupled to a Gaussian, noninteracting environment, the dynamical contribution of the latter is encoded in an object called the influence functional. Here, we relate the influence functional to the process tensor-a more general representation of a quantum stochastic process-describing the evolution. Then, we use this connection to motivate a tensor network algorithm for the simulation of multitime correlations in open systems, building on recent work where the influence functional is represented in terms of time evolving matrix product operators. By exploiting the symmetries of the influence functional, we are able to use our algorithm to achieve orders-of-magnitude improvement in the efficiency of the resulting numerical simulation. Our improved algorithm is then applied to compute exact phonon emission spectra for the spin-boson model with strong coupling, demonstrating a significant divergence from spectra derived under commonly used assumptions of memorylessness.
\end{abstract}

DOI: 10.1103/PhysRevLett.123.240602

Introduction.-All nanoscale quantum systems are open, meaning they inevitably interact with their environments, exchanging energy and generating correlations. If the system and its environment remain approximately uncorrelated, then the reduced system dynamics is well described by a Markovian model [1-3]. However, in physical systems such as photosynthetic complexes, nanoscale lasers, and quantum thermal machines [4-6], the need to go beyond a Markovian description has long been recognized, and techniques accounting for non-Markovian physics have been developed, with a varying breadth of applicability. Analytical methods involving time-local equations of motion exist but tend to be highly restricted to specific parameter regimes [7-9]. Exact simulation often requires numerical methods, e.g., discrete path integrals [10-14], memory kernels [15-21], hierarchical equations of motion $[22,23]$, and others [24-26]. Overall, these tend to scale poorly with both simulation time and system size [27], making them inapplicable to processes involving large complexes or when long time dynamics is important.

Recently, tensor network methods have been applied to the simulation [28-31] and characterization [32,33] of open quantum dynamics. Physically, these methods incorporate the fact that typical open quantum systems are only finitely correlated with their environments, massively reducing their description [34]. In particular, Strathearn et al. [35] reformulated the discrete path integral for open systems with Gaussian environments in terms of matrix product operators; the resulting time-evolving matrix product operator (TEMPO) algorithm is numerically exact and has an efficiency comparable to other state of the art methods. By only considering the most important non-Markovian contributions to the dynamics, the algorithm circumvents the exponential memory scaling of the bare path integral, in a similar spirit to earlier path-filtering techniques [36-38]. Motivated by this success, it is natural to ask if tensor network methods can be efficiently generalized from the simulation of reduced system density operators, to the simulation of general non-Markovian processes and multitime correlations, which typically require many realizations of the dynamics to characterize.

In this Letter, we propose such a generalization, by making a formal connection between the path integral structure and the recently developed process tensor framework for characterizing general non-Markovian quantum processes [32]. Then, we use this to argue for an alternative TEMPO algorithm, where we exploit symmetry in the underlying tensor network to better account for the causal structure inherent in the dynamics. This not only allows efficient computation of multitime correlation functionsthe simulation need only run once to extract all multitime observable properties-but also opens the door to simulating more general models. Our alternative formulation is demonstrated to significantly improve the efficiency of the method, which we use to straightforwardly compute nonMarkovian emission spectra for the spin-boson model, beyond the point where the commonly used quantum regression theorem breaks down [39].

Process tensor framework.-We consider stationary unitary dynamics of system $S$ along with its environment $E$ and suppose $S$ is transformed by superoperators $\mathcal{A}_{j}$ at discrete, evenly spaced times $\left\{t_{k-1}, \ldots, t_{0}\right\}$, with 
$\delta t=t_{j}-t_{j-1}$. In an experiment, these superoperators could correspond to interventions on the system as it evolves, i.e., unitary rotations, measurements, etc., in which case they are completely positive and, if the interventions are not conditional on a particular measurement outcome, trace preserving. Otherwise, they could represent more abstract transformations useful in the computation of quantities such as emission spectra. The reduced, and potentially subnormalized, state of the system at time $t_{k}$ is given by

$$
\rho_{k}\left(\left\{\mathcal{A}_{j}\right\}\right)=\operatorname{tr}_{E}\left\{\mathcal{U}_{\delta t} \mathcal{A}_{k-1} \ldots \mathcal{U}_{\delta t} \mathcal{A}_{0}\left[\chi_{0}\right]\right\}
$$

where $\mathcal{U}_{\delta t}$ is a superoperator representation of the unitary evolution of duration $\delta t$, i.e., $\mathcal{U}_{\delta t}[\rho]=U_{\delta t} \rho U_{\delta t}^{\dagger}$, with $U_{\delta t}$ a unitary matrix, and $\chi_{0}$ is the initial system-environment state. The inclusion of intermediate transformations makes it possible to consider a much broader class of physical properties than free evolution of the density operator would allow.

Since the state at time $t_{k}$ in Eq. (1) is linearly related to each of the superoperators $\mathcal{A}_{j}$, it can be written as a linear function of the tensor product of their Choi representations $\quad \mathbf{A}_{k-1: 0}=\mathrm{A}_{k-1} \otimes \ldots \otimes \mathrm{A}_{1} \otimes \mathrm{A}_{0}$, with $\mathrm{A}_{j}:=\sum_{s r} \mathcal{A}_{j}[|s\rangle\langle r|] \otimes|s\rangle\langle r|$ obtained via the ChoiJamiołkowski isomorphism [40-42]; here, $\{|s\rangle\}$ forms an orthonormal basis for $S$. Specifically, $\rho_{k}\left(\left\{\mathcal{A}_{j}\right\}\right)=$ $\operatorname{tr}_{k-1: 0}\left\{\Upsilon_{k: 0}\left(\mathbb{1}_{k} \otimes \mathbf{A}_{k-1: 0}^{T}\right)\right\}$, with the trace over all subsystems on which $\mathbf{A}_{k-1: 0}$ acts. As we detail in the Supplemental Material [43],

$$
\begin{aligned}
\Upsilon_{k: 0}= & \sum_{\vec{s}^{\prime}, \vec{r}^{\prime}, \vec{s}, \vec{r}} \operatorname{tr}\left\{\mathcal{U}_{\delta t}^{\left(s_{k}^{\prime}, r_{k}^{\prime}, s_{k-1}, r_{k-1}\right)} \ldots \mathcal{U}_{\delta t}^{\left(s_{1}^{\prime}, r_{1}^{\prime}, s_{0}, r_{0}\right)}\left[\chi_{0}^{\left(r_{0}^{\prime}, s_{0}^{\prime}\right)}\right]\right\} \\
& \times\left|s_{k}^{\prime} s_{k-1} \ldots s_{1}^{\prime} s_{0} s_{0}^{\prime}\right\rangle\left\langle r_{k}^{\prime} r_{k-1} \ldots r_{1}^{\prime} r_{0} r_{0}^{\prime}\right|,
\end{aligned}
$$

with environment superoperators $\mathcal{U}_{\delta t}^{\left(s^{\prime}, r^{\prime}, s, r\right)}\left[\rho^{E}\right]=$ $\left\langle s^{\prime}\left|U_{\delta t}\left(|s\rangle\langle r| \otimes \rho^{E}\right) U_{\delta t}^{\dagger}\right| r^{\prime}\right\rangle$ and operators $\chi_{0}^{\left(r^{\prime}, s^{\prime}\right)}=\left\langle r^{\prime}\left|\chi_{0}\right| s^{\prime}\right\rangle$, is the Choi representation of the process tensor [32], a manybody operator (on $2 k+1$ copies of $S$ ) containing all information about the system's evolution that is independent of the superoperators $\left\{\mathcal{A}_{j}\right\}$. Correlations between subsystems of $\Upsilon_{k: 0}$ correspond to temporal correlations between observables, and a representation in terms of process tensors has been shown to consistently generalize stochastic processes, and related notions such as Markov order, to the quantum case [44-47]. The process tensor is illustrated graphically in Fig. 1 and can be thought of as a sequence of correlated maps on the system [48].

Gaussian influence functional.-Here, we consider the specific structure of the process tensor for systems interacting with Gaussian environments, where the systemenvironment Hamiltonian and initial state depend, at most, quadratically on environment creation and annihilation (a)
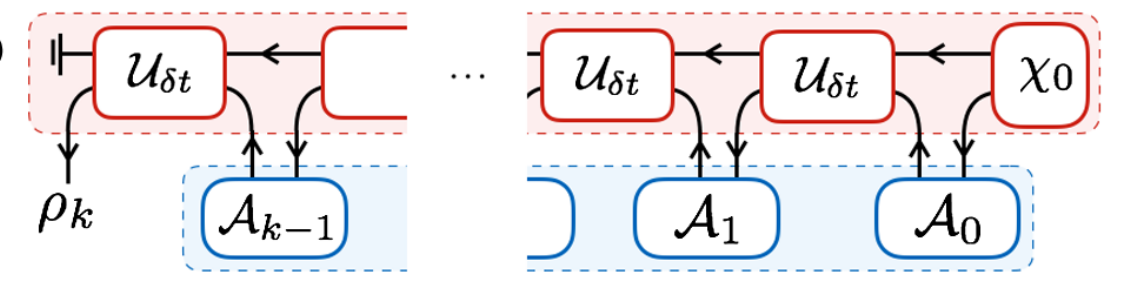

$\Downarrow \quad \delta t \rightarrow 0$

(b)
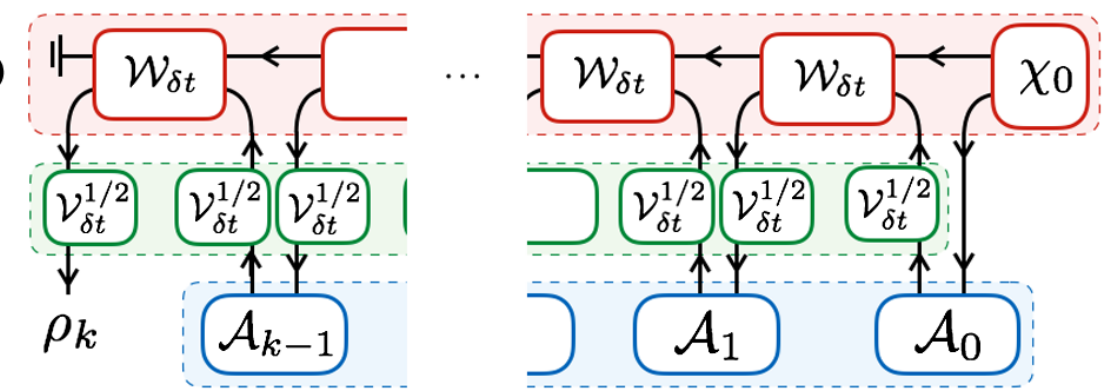

FIG. 1. (a) An arbitrary process with interventions can be represented as a matrix product form tensor network, the process tensor (upper row) that contracts with a filter function consisting of a sequence of superoperators (lower row). This makes it possible to separate implemented control operations from the underlying uncontrolled process. (b) In the infinitesimal time step limit, the uncontrolled process can be further decomposed into free evolution of the system (middle row) and a generalized influence functional capturing the influence of the environment.

operators. For concreteness, we focus on spin-boson type models, but our results would extend to fermionic environments [49] (as well as those with additional commuting linear interaction terms). Working in natural units $\left(\hbar=k_{B}=1\right)$, we consider a spin system, with Hilbert space dimension $d$, interacting linearly with a bosonic bath described by the Hamiltonian $H=H_{0}+H_{B}$. Here, $H_{0}$ describes the free spin system, and the full bath influence is collected in $H_{B}=\hat{s} \sum_{n}\left(g_{n} \hat{a}_{n}+g_{n}^{*} \hat{a}_{n}^{\dagger}\right)+\sum_{n} \omega_{n} \hat{a}_{n}^{\dagger} \hat{a}_{n}$. A bath mode $n$ has energy $\omega_{n}$, is created (annihilated) by the bosonic operator $\hat{a}_{n}^{\dagger}\left(\hat{a}_{n}\right)$, and interacts with $S$ through the operator $\hat{s}$ with coupling strength $g_{n}$. For simplicity, we take the initial state to be product, such that $\chi_{0}=\rho_{0} \otimes \tau_{\beta}$, with the environment initially described by a thermal state $\tau_{\beta}=\exp \left[-\beta \sum \omega_{n} a_{n}^{\dagger} a_{n}\right] / \mathcal{Z}$ at inverse temperature $\beta$, where $\mathcal{Z}=\operatorname{tr}\left\{\exp \left[-\beta \sum \omega_{n} a_{n}^{\dagger} a_{n}\right]\right\}$.

In the limit that the time difference $\delta t$ is small, the generated unitary dynamics can be approximately separated into contributions arising from $H_{0}$ and $H_{B}$ as $\mathcal{U}_{\delta t} \simeq \mathcal{V}_{\delta t}^{1 / 2} \mathcal{W}_{\delta t} \mathcal{V}_{\delta t}^{1 / 2}$, where $\mathcal{V}_{\delta t}$ describes the free dynamics of $S$ and $\mathcal{W}_{\delta t}$ describes the environment influence. The discrepancy between the approximate unitary maps and the actual ones vanishes as $\mathcal{O}\left(\delta t^{3}\right)$ for this symmetric decomposition [50]. Since the Hamiltonian only contains a single interaction term, $\mathcal{W}_{\delta t}$ preserves the eigenbasis of the corresponding system operator $\hat{s}=\sum_{s} \lambda_{s}|s\rangle\langle s|$ : $\left\langle s^{\prime}\left|\mathcal{W}_{\delta t}[|s\rangle\langle r|]\right| r^{\prime}\right\rangle=\delta_{s s^{\prime}} \delta_{r r^{\prime}} \mathcal{W}_{\delta t}^{(s, r)}$. Together with the decomposition of unitary maps, this allows us to approximate the process tensor as $\Upsilon_{k: 0} \simeq\left(\mathcal{V}_{\delta t}^{1 / 2} \otimes \mathcal{V}_{\delta t}^{* 1 / 2}\right)^{\otimes k}\left[\mathcal{F}_{k: 0}\right] \otimes \rho_{0}$, where 


$$
\begin{aligned}
\mathcal{F}_{k: 0}= & \sum_{\vec{s}, \vec{r}} \operatorname{tr}_{E}\left\{\mathcal{W}_{\delta t}^{\left(s_{k}, r_{k}\right)} \ldots \mathcal{W}_{\delta t}^{\left(s_{1}, r_{1}\right)}\left[\tau_{\beta}\right]\right\} \\
& \times\left|s_{k} s_{k} \ldots s_{1} s_{1}\right\rangle\left\langle r_{k} r_{k} \ldots r_{1} r_{1}\right|,
\end{aligned}
$$

is an operator representation of the discretized FeynmanVernon influence functional [51] encoding environment induced correlations.

For Gaussian environments, the bath degrees of freedom can be traced over analytically using standard path integral techniques $[10,11,14,52]$. In this case, introducing the $d^{2}$ compound indices $\alpha=(s, r)$, an element of the influence functional $\mathcal{F}_{k: 0}^{\alpha_{k}, \ldots, \alpha_{1}}:=\left\langle s_{k} s_{k} \ldots s_{1} s_{1}\left|\mathcal{F}_{k: 0}\right| r_{k} r_{k} \ldots r_{1} r_{1}\right\rangle$ can be decomposed as

$$
\mathcal{F}_{k: 0}^{\alpha_{k}, \ldots, \alpha_{1}}=\prod_{i=1}^{k} \prod_{j=1}^{i}\left[b_{(i-j)}\right]^{\alpha_{i}, \alpha_{j}},
$$

where $b_{(i-j)}$ is called an influence tensor; the exact form, which can often be approximated by an analytic function [53,54], is given in the Supplemental Material [43] along with a full derivation of Eqs. (3) and (4). The influence tensors connect the dynamics around time step $i$ with that around step $j$, quantifying temporal correlations mediated by the environment between those two points; that is, they describe memory effects. Since the Hamiltonian is timeindependent, the individual tensors $\left[b_{l}\right]$ depend only on the temporal separation $l \delta t$, simplifying the complexity considerably. However, since the influence functional is a $k$ index tensor, it is still potentially exponentially complex; we now show how viewing Eq. (4) as a tensor network can make its calculation more tractable.

Tensor network simulation.-In many cases, the environment interaction produces only finite length correlations in $\mathcal{F}_{k: 0}$, a fact used by the authors of Ref. [35] to circumvent the exponential complexity growth by representing it in terms of matrix product operators (MPOs) [55]. To introduce this representation, we extend our two-index influence tensors to three-index tensors as $\left[b_{(i-j)}\right]^{\gamma, \alpha_{i}}{ }_{\alpha_{j}}:=$ $\delta_{\alpha_{j}}^{\gamma}\left[b_{(i-j)}\right]_{\alpha_{j}}^{\alpha_{i}}$, where, by convention, upper and lower repeated indices are summed over (otherwise, tensor elements differing only through raising or lowering are equal). In terms of these, we define the nonlocal timeevolving MPOs

$$
\begin{aligned}
\mathcal{F}_{k: 0}^{\alpha_{k} \ldots \alpha_{1}} & =\prod_{i=1}^{k}\left[b_{0}\right]_{\beta_{1}}^{\alpha_{i}} \prod_{j=1}^{i-2}\left[b_{j}\right]_{\beta_{j+1}}^{\beta_{j}, \alpha_{i-j}}\left[b_{i-1}\right]^{\beta_{i-1}, \alpha_{1}} \\
& =\prod_{i=1}^{k} 0-1 \alpha^{\wedge}-\stackrel{1}{\alpha_{i}},
\end{aligned}
$$

where the outgoing (ingoing) arrows in the graphical representation indicate upper (lower) indices, and lines running through the row and column of a given " 0 " tensor are fixed to have the same index through Kronecker deltas; superfluous indices are traced over at the boundaries. Then, (a) Nonlocal network boundary

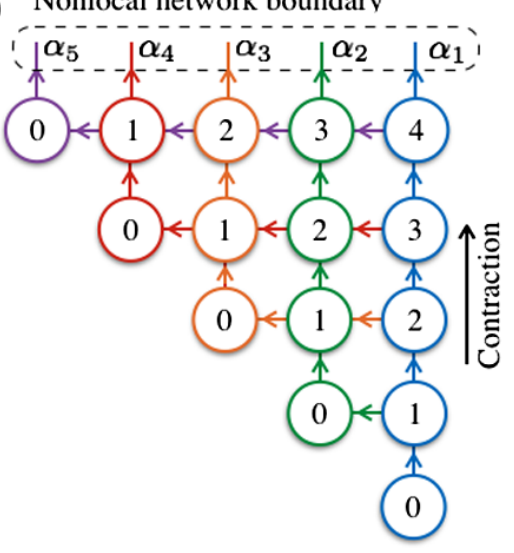

(b) $\left.\left.\left.\left.\left.\right|^{\alpha_{5}}\right|^{\alpha_{4}}\right|^{\alpha_{3}}\right|^{\alpha_{2}}\right|^{\alpha_{1}}$
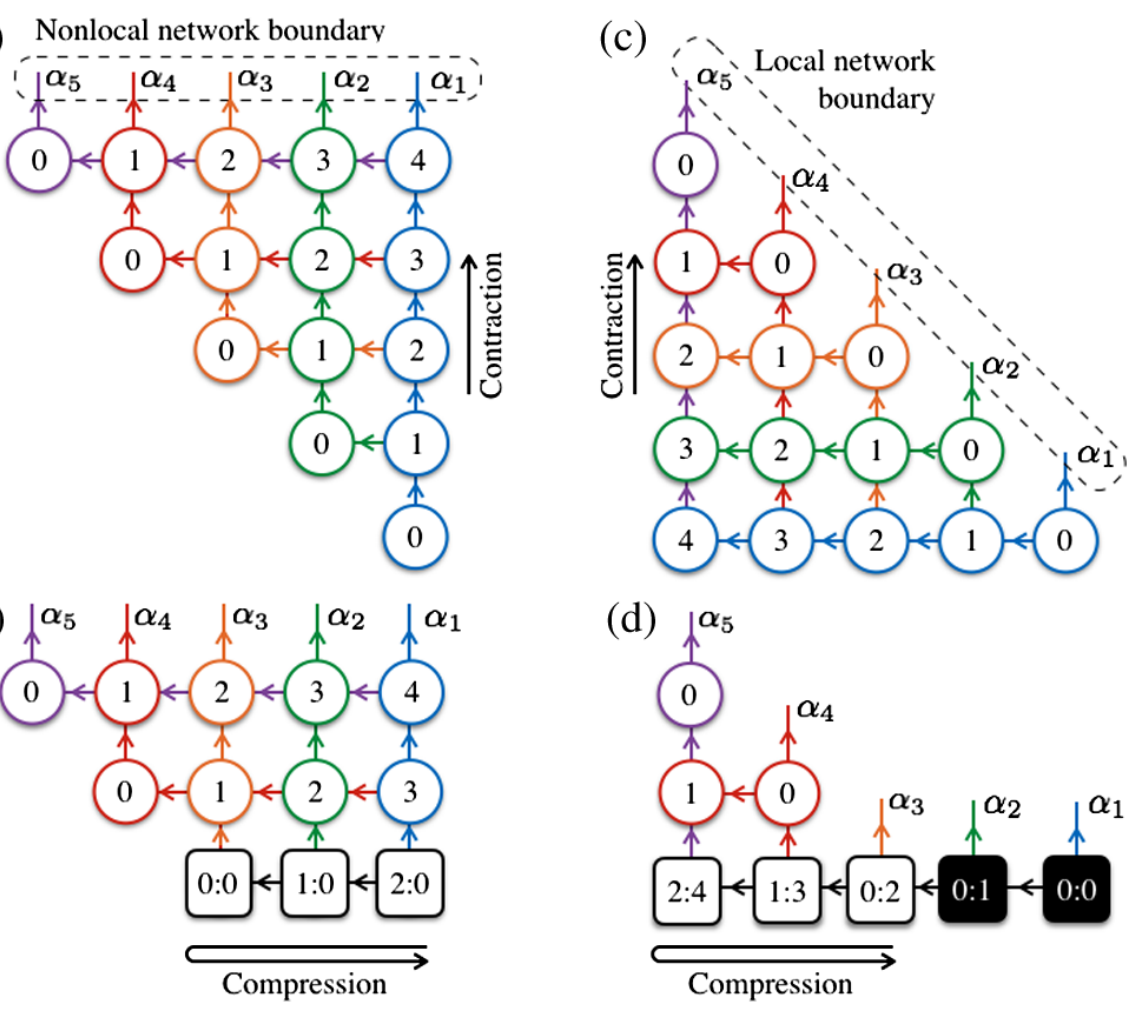

(d) $\lambda^{\alpha_{5}}$

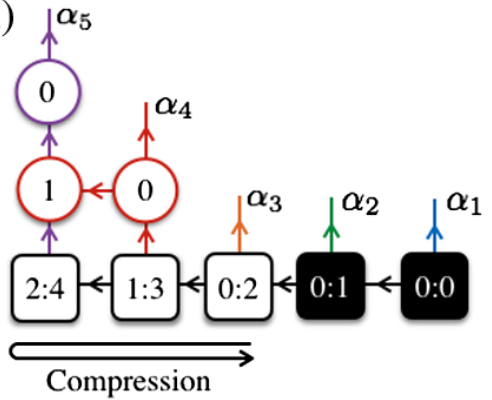

FIG. 2. Tensor network representation of the influence functional on five time steps, with nodes representing influence tensors and labeled by time step separation. Before contraction, indices are constrained to be equal along rows and columns in the network; therefore, the open boundary can be shifted to any tensor in the same column [panels (a) and (b)] or row [panels (c) and (d)]. (a) With the nonlocal boundary choice of Ref. [35], free indices are attached to influence tensors encoding memory effects over all different timescales. (b) The network is contracted iteratively from below, row by row, down to a boundary MPO. (c) With the local boundary choice, the free indices are always attached to time-local influence tensors. (d) Contraction proceeds as indicated, with the causal influence of each open leg sequentially incorporated into the wider network. The influence functional on an open leg is fixed once the corresponding layer has been contracted over.

the full influence functional can be constructed by iteratively multiplying such MPOs. If we label individual MPOs in the product by $G^{\alpha_{i}, \ldots, \alpha_{1}}$, then we can express the iterative multiplication as $\mathcal{F}_{k: 0}^{\alpha_{k}, \ldots, \alpha_{1}}=\mathcal{G}_{\beta_{k-1}, \ldots, \beta_{1}}^{\alpha_{k}, \alpha_{k-1}, \ldots, \alpha_{1}} \mathcal{F}_{k-1: 0}^{\beta_{k-1}, \ldots, \beta_{1}}$, with $\mathcal{G}_{\beta_{k-1}, \ldots, \beta_{1}}^{\alpha_{k}, \alpha_{k-1}, \ldots, \alpha_{1}}:=G^{\alpha_{k}, \ldots, \alpha_{1}} \delta_{\beta_{k-1}}^{\alpha_{k-1}} \ldots \delta_{\beta_{1}}^{\alpha_{1}} ;$ this is represented graphically by the two-dimensional tensor network shown in Fig. 2(a). Conceptually, the use of time-evolving MPOs, allows the state of the system to be propagated by updating indices to encode memory effects from the past process. This type of propagation is analogous to a description in terms of a time nonlocal memory kernel, since open legs are connected to tensors describing the influence of the state at various points in its history [56].

A key insight of this Letter is that the decomposition of the influence functional into MPOs is not unique. Kronecker deltas implicit in Eq. (5) mean that each open leg in Fig. 2 could be shifted to any tensor in the same row or column. In particular, the causal structure of the process tensor motivates an alternative definition in terms of local time-evolving MPOs 


$$
\begin{aligned}
& \mathcal{F}_{k: 0}^{\alpha_{k}, \ldots, \alpha_{1}}=\prod_{i=1}^{k}\left[b_{k-i}\right]_{\beta_{k-i}}^{\alpha_{k}} \prod_{j=1}^{k-i-1}\left[b_{j}\right]_{\beta_{j}}^{\beta_{j+1}, \alpha_{j+1}}\left[b_{0}\right]^{\beta_{1}, \alpha_{i}} \\
& =\prod_{i=1}^{k} \stackrel{\lambda^{\alpha_{k}}}{k-i}+\stackrel{1}{-1}+\stackrel{\lambda^{\alpha}}{-},
\end{aligned}
$$

where, now, we end up with a redundant index at the left boundary, which we trace over, and at the right boundary we impose the condition that the lower index must equal $\alpha_{i}$. Labeling the individual MPOs in the product by $C^{\alpha_{k}, \ldots, \alpha_{i}}$, the iterative multiplication can be expressed as $\tilde{\mathcal{F}}_{(k: i+1)}^{\alpha_{k}, \ldots, \alpha_{1}}:=\mathcal{C}_{\beta_{k}, \ldots, \beta_{i+1}}^{\alpha_{k}, \ldots, \alpha_{i+1}} \tilde{\mathcal{F}}_{(k: i)}^{\beta_{k}, \ldots, \beta_{i+1} \alpha_{i}, \ldots, \alpha_{1}}$, with $\quad \mathcal{C}_{\gamma_{k}, \ldots, \gamma_{i}}^{\alpha_{k}, \ldots, \alpha_{i}}:=$ $C^{\alpha_{k}, \ldots, \alpha_{i}} \delta_{\gamma_{k}}^{\alpha_{k}} \ldots \delta_{\gamma_{i}}^{\alpha_{i}}$ (for $i \geq 1$ ) and $\tilde{\mathcal{F}}_{(k: 1)}^{\alpha_{k}, \ldots, \alpha_{1}}:=C^{\alpha_{k}, \ldots, \alpha_{1}}$. The resulting network representation for the influence functional $\mathcal{F}_{k: 0}=\tilde{\mathcal{F}}_{(k: k)}$ is shown in Fig. 2(c). Conceptually, the local time-evolving MPOs propagate the state by updating a set of effective memory space indices that describe how $E$ is conditioned by $S$. Since the process tensor, and hence, the influence functional, has a welldefined causal structure, this conditioning only occurs from the past to the future. This means that, for a fixed evolution time, the size of the tensor to be updated decreases with each iteration.

In contracting the network, efficiency is achieved by incorporating a tensor compression procedure of the obtained boundary in each iteration. In this Letter, we make use of the singular value compression procedure (see Supplemental Material [43]) [35,55]. The local tensors at the boundary are subjected to a singular value decomposition, and singular values below a specified cutoff $\lambda_{c}$ are discarded. For the nonlocal algorithm [Figs. 2(a) and 2(b)], the tensors contracted in each iteration encode information about the influence of multiple time steps on each other. When correlations become smaller at longer time scales, as is typical, not all this information is dynamically relevant. The local algorithm [Figs. 2(c) and 2(d)] incorporates this insight and separates out the most important contribution by including only the future influence of the environment at each time step. Generally, the most local contributions have the largest singular values, and therefore, the separation means that the part of the boundary being propagated in the local case is less correlated, translating into a more efficient algorithm.

Network complexity for a two level system.-Now, we turn to the specific simulation of the dynamics of a twolevel system and compare the performance of the nonlocal and local algorithms. Consider the free Hamiltonian $H_{0}=$ $\Omega \sigma_{x} / 2$ and $\hat{s}=\sigma_{z} / 2$, where $\sigma_{x}$ and $\sigma_{z}$ are the usual Pauli operators. The environment is fully characterized by its spectral density defined as $J(\omega)=\sum_{n}\left|g_{n}\right|^{2} \delta\left(\omega-\omega_{n}\right)$ [1]. Here, we consider a continuum bath model with spectral density $J(\omega)=\left(\alpha \omega_{c} / 2\right)\left(\omega / \omega_{c}\right)^{\nu} \exp \left(-\omega / \omega_{c}\right)$ with coupling strength $\alpha$, cutoff frequency $\omega_{c}$ and Ohmicity $\nu$, where, for an Ohmic spectral density, $\nu=1$.

The computational complexity is quantified by the computation time required to contract the network with a fixed singular value cutoff (see Supplemental Material [43] for details). In general, this will depend on the overall magnitude of the influence functional as well as the characteristic memory time quantifying how elements of the influence tensors $b_{(i-j)}$ decrease in magnitude at large $|i-j|$. In the Supplemental Material [43], we show that, for fixed evolution time, the memory time goes as $\alpha /\left(\beta \omega_{c}\right)$ when $\omega_{c}$ is large, and that the total hardness goes as $\alpha \omega_{c} t_{\max }^{2} / \beta$ when $\omega_{c}$ is small. In Fig. 3, we plot the computation time for the local and nonlocal algorithms as a function of coupling strength and bath timescale. We find that the local representation outperforms the nonlocal one by 1 to 2 orders of magnitude, and that the improvement increases at larger coupling strengths (In the Supplemental Material [43], we show that the advantage
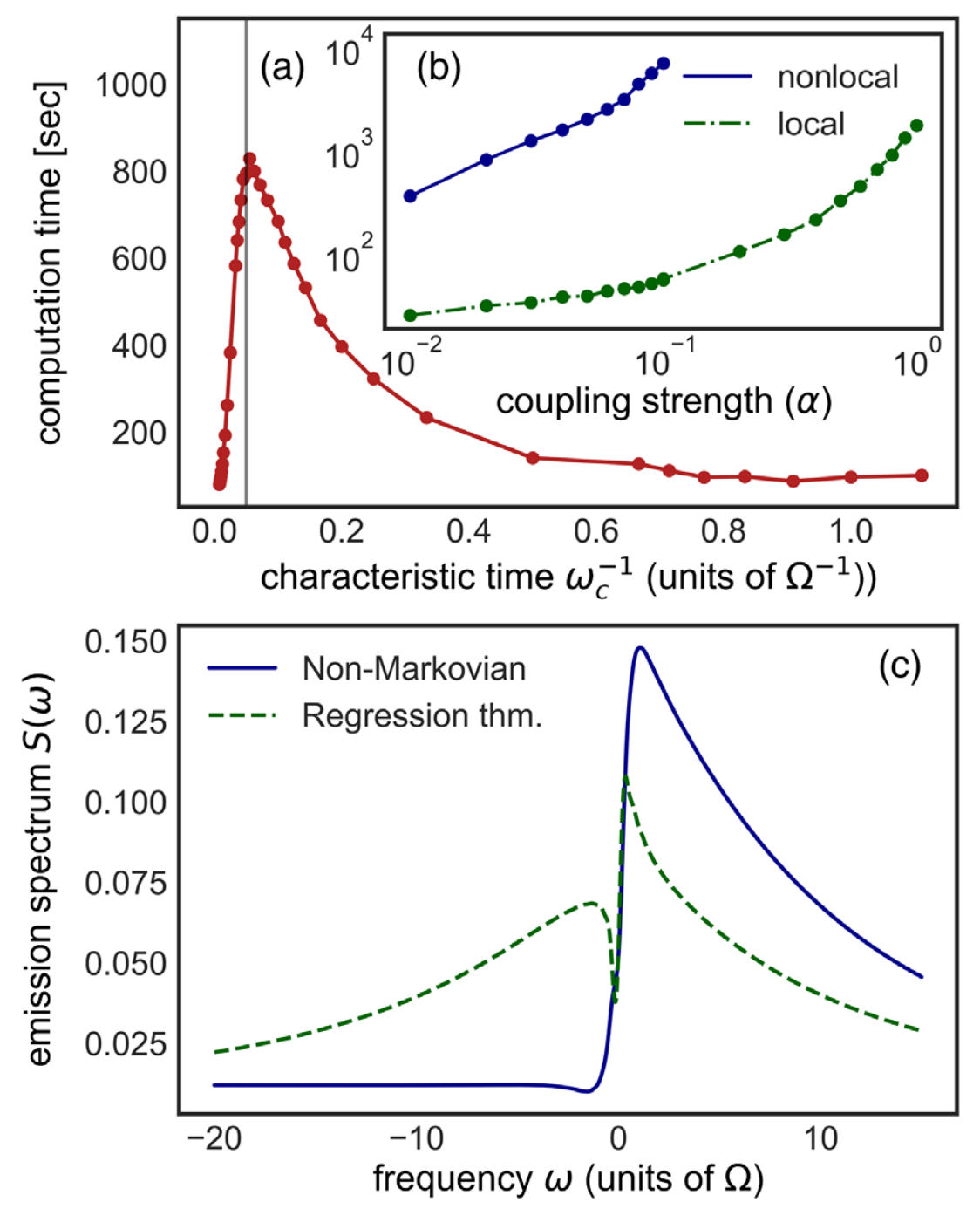

FIG. 3. (a) Variation of computation time with the inverse of the cutoff frequency for the local algorithm at a coupling strength of $\alpha=0.7$, for an Ohmic spectral density with $T=0.01 \Omega$ and $\lambda_{c}=10^{-6}$, the vertical line shows the (fixed) time step size used for the Trotter-Suzuki decomposition. (b) Comparison between the computation time of the nonlocal [Eq. (5)] and local [Eq. (6)] time-evolving MPO algorithms as a function of coupling strength with $\omega_{c}=10 \Omega$ (the remaining parameters are unchanged). (c) Steady state phonon emission spectrum at $\alpha=0.5$, the (numerically converged) non-Markovian spectrum is simulated using the local algorithm and is compared with the spectrum obtained using the regression theorem. 
persists into the easier weak coupling regime and for varying $\omega_{c}$.).

It should be kept in mind that, unlike in most simulation methods, including the original TEMPO algorithm, the object we are computing is the full process tensor, which efficiently encodes all multitime properties of the system. In particular, we can compute the steady state emission spectrum $\quad S(\omega)=\operatorname{Re}\left\{\int_{0}^{\infty} d \tau\left[g^{(1)}(\tau)-g^{(1)}(\infty)\right] e^{-i \omega \tau}\right\}$, defined in terms of the two-point correlation function $g^{(1)}(\tau)=\lim _{t \rightarrow \infty}\left\langle\sigma^{\dagger}(t+\tau) \sigma(t)\right\rangle$, with $\sigma^{\dagger}$ and $\sigma$ the raising and lowering operators on the spin system. To compute it, we take all superoperators which the process tensor acts on to be the identity $\mathcal{I}$ (with action $\mathcal{I}[\rho]=\rho$ ) except for two, which append a raising or lowering operator, respectively. In Fig. 3(c), we study the physical effects of systemenvironment correlations by looking at the phonon emission spectrum. We compare this with the spectrum computed using the quantum regression theorem, which approximates intermediate dynamics with that from an initial product state and is valid in the weak-coupling limit [5]. The regression theorem correlations are obtained by breaking all correlations in the process tensor across time steps at which the raising and lowering operators are evaluated. Figure 3(c) shows that non-Markovian effects produce a phonon sideband in the spectrum only at positive frequencies, this is contrasted with the regression theorem result which also gives emission at negative frequencies. These significant differences illustrate the importance of accounting for non-Markovian physics.

Conclusion.-In this Letter, we have established a direct connection between the process tensor framework for characterizing non-Markovian quantum processes and the path integral formulation of open quantum dynamics. By relating the influence functional to a process tensor on an infinitesimal time grid, which has an explicit causal structure, we were able to build on recent progress in the simulation of open quantum systems in terms of tensor networks. Specifically, we showed that the speed of the TEMPO algorithm, when computing the multitime properties encapsulated in the process tensor, can be improved by orders of magnitude by shifting the corresponding tensor network boundary from a temporally nonlocal to a local one. Our contribution is immediately applicable to the efficient simulation of nontrivial spectral phenomena in realistic complex open systems.

The utility of the nonlocal TEMPO algorithm has been illustrated by computing the Ohmic localization transition, and the dynamics of complex problems with multiple separated timescales [35]. The improved algorithm presented here is capable of exploring the same physics more efficiently and easily extends to the computation of multitime observables, of the sort crucial to describing, for example, ultrafast spectroscopy experiments [57]. Moreover, relating open systems techniques, such as the path integral, to the more general process tensor formalism indicates how they might be adapted to more complex system-environment interactions, or even beyond the Gaussian regime. Even within the spin-boson model, the freedom of boundary choice we have identified could be further exploited in other contexts. While the local choice appears optimal here, it may be that, for other, structured spectral densities, different boundary choices are more efficient, a point whose exploration we leave for future work.

F. A. P. would like to thank A. Nazir, for asking one of the questions that motivated this work, and A. Strathearn, B. W. Lovett, and P. Kirton for introducing and explaining the use of the time-evolving matrix product operator algorithm. M. R. J. was supported by the Independent Research Fund Denmark.

*matrj@fysik.dtu.dk

†felix.pollock@monash.edu

[1] H.-P. Breuer and F. Petruccione, The Theory of Open Quantum Systems (Oxford University Press, Oxford, 2002).

[2] U. Weiss, Quantum Dissipative Systems (World Scientific, Singapore, 2012).

[3] H. J. Carmichael, Statistical Methods in Quantum Optics 1: Master Equations and Fokker-Planck Equations (Springer, New York, 2003).

[4] C. A. Mujica-Martinez, P. Nalbach, and M. Thorwart, Quantification of non-Markovian effects in the FennaMatthews-Olson complex, Phys. Rev. E 88, 062719 (2013).

[5] D. P. S. McCutcheon, Optical signatures of non-Markovian behavior in open quantum systems, Phys. Rev. A 93, 022119 (2016).

[6] D. Newman, F. Mintert, and A. Nazir, Performance of a quantum heat engine at strong reservoir coupling, Phys. Rev. E 95, 032139 (2017).

[7] B. Vacchini and H.-P. Breuer, Exact master equations for the non-Markovian decay of a qubit, Phys. Rev. A 81, 042103 (2010).

[8] C. K. Lee, J. Moix, and J. Cao, Accuracy of second order perturbation theory in the polaron and variational polaron frames, J. Chem. Phys. 136, 204120 (2012).

[9] A. Fruchtman, N. Lambert, and E. M. Gauger, When do perturbative approaches accurately capture the dynamics of complex quantum systems?, Sci. Rep. 6, 28204 (2016).

[10] N. Makri and D. E. Makarov, Tensor propagator for iterative quantum time evolution of reduced density matrices. I. Theory, J. Chem. Phys. 102, 4600 (1995).

[11] N. Makri and D. E. Makarov, Tensor propagator for iterative quantum time evolution of reduced density matrices. II. Numerical methodology, J. Chem. Phys. 102, 4611 (1995).

[12] P. Nalbach, A. Ishizaki, G. R. Fleming, and M. Thorwart, Iterative path-integral algorithm versus cumulant time-nonlocal master equation approach for dissipative biomolecular exciton transport, New J. Phys. 13, 063040 (2011).

[13] N. S. Dattani, Numerical Feynman integrals with physically inspired interpolation: Faster convergence and significant 
reduction of computational cost, AIP Adv. 2, 012121 (2012).

[14] A. Strathearn, B. W. Lovett, and P. Kirton, Efficient realtime path integrals for non-Markovian spin-boson models, New J. Phys. 19, 093009 (2017).

[15] Q. Shi and E. Geva, A new approach to calculating the memory kernel of the generalized quantum master equation for an arbitrary system-bath coupling, J. Chem. Phys. 119, 12063 (2003).

[16] G. Cohen and E. Rabani, Memory effects in nonequilibrium quantum impurity models, Phys. Rev. B 84, 075150 (2011).

[17] J. Cerrillo and J. Cao, Non-Markovian Dynamical Maps: Numerical Processing of Open Quantum Trajectories, Phys. Rev. Lett. 112, 110401 (2014).

[18] R. Rosenbach, J. Cerrillo, S. F. Huelga, J. Cao, and M. B. Plenio, Efficient simulation of non-Markovian system-environment interaction, New J. Phys. 18, 023035 (2016).

[19] M. Buser, J. Cerrillo, G. Schaller, and J. Cao, Initial systemenvironment correlations via the transfer-tensor method, Phys. Rev. A 96, 062122 (2017).

[20] A. Gelzinis, E. Rybakovas, and L. Valkunas, Applicability of transfer tensor method for open quantum system dynamics, J. Chem. Phys. 147, 234108 (2017).

[21] F. A. Pollock and K. Modi, Tomographically reconstructed master equations for any open quantum dynamics, Quantum 2, 76 (2018).

[22] Y. Tanimura, Stochastic Liouville, Langevin, FokkerPlanck, and master equation approaches to quantum dissipative systems, J. Phys. Soc. Jpn. 75, 082001 (2006).

[23] J. Strümpfer and K. Schulten, Open quantum dynamics calculations with the hierarchy equations of motion on parallel computers, J. Chem. Theory Comput. 8, 2808 (2012).

[24] R. Bulla, T. A. Costi, and T. Pruschke, Numerical renormalization group method for quantum impurity systems, Rev. Mod. Phys. 80, 395 (2008).

[25] H.-T. Chen, G. Cohen, and D. R. Reichman, Inchworm Monte Carlo for exact non-adiabatic dynamics. I. Theory and algorithms, J. Chem. Phys. 146, 054105 (2017).

[26] H.-T. Chen, G. Cohen, and D. R. Reichman, Inchworm Monte Carlo for exact non-adiabatic dynamics. II. Benchmarks and comparison with established methods, J. Chem. Phys. 146, 054106 (2017).

[27] I. de Vega and D. Alonso, Dynamics of non-Markovian open quantum systems, Rev. Mod. Phys. 89, 015001 (2017).

[28] A. W. Chin, Á. Rivas, S. F. Huelga, and M. B. Plenio, Exact mapping between system-reservoir quantum models and semi-infinite discrete chains using orthogonal polynomials, J. Math. Phys. (N.Y.) 51, 092109 (2010).

[29] J. Prior, A. W. Chin, S. F. Huelga, and M. B. Plenio, Efficient Simulation of Strong System-Environment Interactions, Phys. Rev. Lett. 105, 050404 (2010).

[30] F. A. Y. N. Schröder and A. W. Chin, Simulating open quantum dynamics with time-dependent variational matrix product states: Towards microscopic correlation of environment dynamics and reduced system evolution, Phys. Rev. B 93, 075105 (2016).

[31] M. L. Wall, A. Safavi-Naini, and A. Maria Rey, Simulating generic spin-boson models with matrix product states, Phys. Rev. A 94, 053637 (2016).
[32] F. A. Pollock, C. Rodríguez-Rosario, T. Frauenheim, M. Paternostro, and K. Modi, Non-Markovian quantum processes: Complete framework and efficient characterization, Phys. Rev. A 97, 012127 (2018).

[33] I. A. Luchnikov, S. V. Vintskevich, H. Ouerdane, and S. N. Filippov, Simulation Complexity of Open Quantum Dynamics: Connection with Tensor Networks, Phys. Rev. Lett. 122, 160401 (2019).

[34] R. Orús, A practical introduction to tensor networks: Matrix product states and projected entangled pair states, Ann. Phys. (Amsterdam) 349, 117 (2014).

[35] A. Strathearn, P. Kirton, D. Kilda, J. Keeling, and B. W. Lovett, Efficient non-Markovian quantum dynamics using time-evolving matrix product operators, Nat. Commun. 9, 3322 (2018).

[36] E. Sim and N. Makri, Filtered propagator functional for iterative dynamics of quantum dissipative systems, Comput. Phys. Commun. 99, 335 (1997).

[37] E. Sim, Quantum dynamics for a system coupled to slow baths: On-the-fly filtered propagator method, J. Chem. Phys. 115, 4450 (2001).

[38] N.S. Dattani, FeynDyn: A MATLAB program for fast numerical Feynman integral calculations for open quantum system dynamics on GPUs, Comput. Phys. Commun. 184, 2828 (2013).

[39] L. Li, M. J. W. Hall, and H. M. Wiseman, Concepts of quantum non-Markovianity: A hierarchy, Phys. Rep. 759, 1 (2018).

[40] S. Milz, F. A. Pollock, and K. Modi, An introduction to operational quantum dynamics, Open Syst. Inf. Dyn. 24, 1740016 (2017).

[41] M. A. Nielsen and I. L. Chuang, Quantum Computation and Quantum Information (Cambridge University Press, Cambridge, England, 2011).

[42] M. Wilde, Quantum Information Theory, 2nd ed. (Cambridge University Press, Cambridge, England, 2017).

[43] See Supplemental Material at http://link.aps.org/ supplemental/10.1103/PhysRevLett.123.240602 for an extended derivation of our main results and details of our numerical simulations.

[44] S. Milz, F. Sakuldee, F. A. Pollock, and K. Modi, Kolmogorov extension theorem for (quantum) causal modelling and general probabilistic theories, arXiv:1712.02589.

[45] F. A. Pollock, C. Rodríguez-Rosario, T. Frauenheim, M. Paternostro, and K. Modi, Operational Markov Condition for Quantum Processes, Phys. Rev. Lett. 120, 040405 (2018).

[46] P. Taranto, F. A. Pollock, S. Milz, M. Tomamichel, and K. Modi, Quantum Markov Order, Phys. Rev. Lett. 122, 140401 (2019).

[47] P. Taranto, S. Milz, F. A. Pollock, and K. Modi, Structure of quantum stochastic processes with finite Markov order, Phys. Rev. A 99, 042108 (2019).

[48] F. Sakuldee, S. Milz, F. A. Pollock, and K. Modi, NonMarkovian quantum control as coherent stochastic trajectories, J. Phys. A 51, 414014 (2018).

[49] A. Atland and B. Simon, Condensed Matter Field Theory (Cambridge University Press, Cambridge, England, 2010).

[50] H. F. Trotter, On the product of semi-groups of operators, Proc. Am. Math. Soc. 10, 545 (1959). 
[51] R. P. Feynman and F. L. Vernon, The theory of a general quantum system interacting with a linear dissipative system, Ann. Phys. (N.Y.) 24, 118 (1963).

[52] L. Diósi and L. Ferialdi, General Non-Markovian Structure of Gaussian Master and Stochastic Schrödinger Equations, Phys. Rev. Lett. 113, 200403 (2014).

[53] N. S. Dattani, F. A. Pollock, and D. M. Wilkins, Analytic influence functionals for numerical Feynman integrals in most open quantum systems, Quantum Phys. Lett. 1, 35 (2012), http://www.naturalspublishing.com/download.asp? ArtcID $=407$.
[54] M. Abramowitz and I. A. Stegun, Handbook of Mathematical Functions (Dover Publications, New York, 1964).

[55] U. Schollwöck, The density-matrix renormalization group in the age of matrix product states, Ann. Phys. (Amsterdam) 326, 96 (2011).

[56] This correspondence is not precise, however, and our usage of "(non)local" should not be confused with that in the context of memory kernel convolution.

[57] J. Yuen-Zhou, J. J. Krich, I. Kassal, A. S. Johnson, and A. Aspuru-Guzik, Ultrafast Spectroscopy (IOP, Bristol, 2014). 